\title{
JOINT MULTIUSER DETECTION AND CANCELATION OF NONLINEAR DISTORTION EFFECTS FOR THE UPLINK OF MC-CDMA SYSTEMS
}

\author{
Paulo Silva \\ EST, Univ. of Algarve \\ Faro, Portugal
}

\section{ABSTRACT}

In this paper we consider the uplink transmission in MCCDMA systems (MultiCarrier Coded Division Multiple Access). To reduce the envelope fluctuations of the transmitted signals, the MC-CDMA signal associated to each MT (Mobile Terminal) is submitted to a clipping device,followed by a frequency-domain filtering operation. We define an iterative receiver that jointly performs the MUD (MultiUser Detection) and the estimation and cancelation of the nonlinear distortion effects that are inherent to the transmitted signals.

Our performance results show that the proposed receiver structures allow good performances, even for severely timedispersive channels and/or when a low-PMEPR is intended for each MT. ${ }^{1}$

\section{INTRODUCTION}

MC-CDMA schemes (MultiCarrier Coded Division Multiple Access) [1] combine a CDMA scheme with OFDM modulation (Orthogonal Frequency Division Multiplexing) [2], so as to allow high transmission rates over severe time-dispersive channels without the need of complex receiver implementations. Since the spreading is made in frequency domain, the time synchronization requirements are much lower than with conventional direct sequence CDMA schemes. Moreover, the diversity effect inherent to the spreading allows good uncoded performances, as well as good performances with high code rates. For these reasons, MC-CDMA schemes are good candidates for future broadband wireless systems.

However, since transmission over severely time-dispersive channels destroys the orthogonality between spreading codes, an FDE (Frequency-Domain Equalizer) is required before the de-spreading operation [3, 4]. To avoid significant noise enhancement for channels with deep in-band notches, the FDE is usually optimized under an MMSE criterion (Minimum MeanSquared Error) [3, 4]. However, since an MMSE FDE does not perform an ideal channel inversion, we are not able to fully orthogonalize the different spreading codes of an MC-CDMA signal. This means that we can have severe interference levels especially for fully loaded systems and/or when different powers are assigned to different spreading codes.

A promising non-linear frequency-domain receiver for multicode MC-CDMA signals was proposed in [5]. That receiver, which is based on the IB-DFE (Iterative block Decision Feedback Equalizer), proposed for block transmission with singlecarrier modulations $[6,7]$, allows significant performance im-

${ }^{1}$ This work was partially supported by the FCT project POSI/CPS/46701/2002 - MC-CDMA, the FCT/POCI 2010 research grant SFRH / BD / 24520 / 2005 and the European IST project C-MOBILE.

\author{
Rui Dinis \\ ISR-IST, Tech. Univ. of Lisbon \\ Lisbon, Portugal
}

provements as we increase the number of iterations, especially for fully loaded systems.

As with other multicarrier schemes, the MC-CDMA signals have strong envelope fluctuations and high PMEPR values (Peak-to-Mean Envelope Power Ratio), which lead to amplification difficulties. For this reason, it is desirable to reduce the envelope fluctuations of the transmitted signals. This is particularly important for the uplink transmission, since an efficient, low-cost power amplification is desirable at the MTs (Mobile Terminals). Several techniques have been recommended for reducing the envelope fluctuations of multicarrier signals [8][11]. A promising approach is to employ clipping techniques, combined with a frequency-domain filtering so as to reduce the envelope fluctuations of the transmitted signals while maintaining the spectral occupation of conventional schemes [11]. However, the nonlinear distortion effects can be severe when a low-PMEPR transmission is intended $[11,12]$. To improve the performances, we can employ the receivers proposed in $[13,14]$ where the nonlinear distortion effects are iteratively estimated and compensated.

In this paper we consider the uplink transmission in MCCDMA systems. To allow an efficient power amplification, the PMEPR-reducing techniques of [11] are adopted by each MT. The BS (Base Station) has several receive antennas, so as to reduce the transmit power requirements of each MT. We define an iterative receiver that jointly performs the MUD (MultiUser Detection) and the estimation and cancelation of the nonlinear distortion effects that are inherent to the transmitted signals.

This paper is organized as follows: the transmitter structure considered in this paper is described in sec. II.. In sec. III. we describe the iterative receivers proposed in this paper. Sec. IV. presents a set of performance results and sec. V. is concerned with the conclusions of the paper.

\section{Transmitter StRUCTURE}

In this paper we consider the uplink transmission in MCCDMA systems employing frequency-domain spreading. The frequency-domain block to be transmitted by the $p$ th MT is $\left\{S_{k, p} ; k=0,1, \ldots, N-1\right\}$, where $N=K M$, with $K$ denoting the spreading factor and $M$ the number of data symbols for that MT. The frequency-domain symbols are given by $S_{k, p}=\xi_{p} C_{k, p} A_{k} \operatorname{Mod} M, p$, where $\xi_{p}$ is an appropriate weighting coefficient that accounts for the propagation losses, $\left\{A_{k, p} ; k=0,1, \ldots, M-1\right\}$ is the block of data symbols associated to the $p$ th MT and $\left\{C_{k, p} ; k=0,1, \ldots, N-1\right\}$ is the corresponding spreading sequence ${ }^{2}$ (a pseudo-random spread-

\footnotetext{
${ }^{2}$ This corresponds to uniformly spread the chips associated to a given symbol within the transmission band, i.e., to employ a rectangular interleaver with
} 


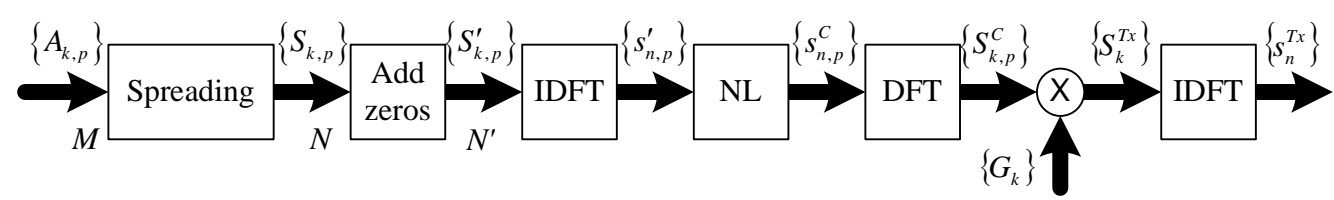

Figure 1: Transmitter model considered in this paper.

ing is assumed, with $C_{k, p}$ belonging to a QPSK constellation; without loss of generality, it is assumed that $\left|C_{k, p}\right|=1$ ).

To reduce the envelope fluctuations of the transmitted signals, we employ the transmitter structure depicted in fig. 1, which is based on the nonlinear signal processing schemes proposed in [11] for reducing the PMEPR of OFDM signals while maintaining the spectral efficiency of conventional OFDM schemes. Within that transmitter, $N^{\prime}-N$ zeros are added to the original frequency-domain block (i.e., $N^{\prime}-N$ idle subcarriers), followed by an IDFT operation so as to generate a sampled version of the time-domain MC-CDMA signal, with an oversampling factor $M_{T x}=N^{\prime} / N$. Each time-domain sample is submitted to a nonlinear device corresponding to an ideal envelope clipping, so as to reduce the envelope fluctuations on the transmitted signal. The clipped signal is then submitted to a frequency-domain filtering procedure, through the set of multiplying coefficients $G_{k}, k=0,1, \ldots, N^{\prime}-1$, in order to reduce the out-of-band radiation levels inherent to the nonlinear operation.

It is shown in [12] that the frequency-domain block to be transmitted by the $p$ th MT is $\left\{S_{k, p}^{T x}=S_{k, p}^{C} G_{k} ; k=\right.$ $\left.0,1, \ldots, N^{\prime}-1\right\}$ can be decomposed into useful and nonlinear self-interference components:

$$
S_{k, p}^{T x}=\alpha_{p} S_{k, p} G_{k}+D_{k, p} G_{k}
$$

with $\alpha_{p}$ defined in $[11,12]$. Throughout this paper we assume that $G_{k}=1$ for the $N$ in-band subcarriers and 0 for the $N^{\prime}-N$ out-of-band subcarriers. In this case

$$
S_{k, p}^{T x}= \begin{cases}\alpha_{p} S_{k, p}+D_{k}, & k \text { in band } \\ 0, & k \text { out of band }\end{cases}
$$

It can be shown that $D_{k, p}$ is approximately Gaussiandistributed, with zero mean; moreover, $E\left[D_{k, p} D_{k^{\prime}, p}^{*}\right]$ can be computed analytically, as described in $[11,12]$.

\section{RECEIVER STRUCTURE}

\section{A. Linear Transmitters}

As usual, it is assumed that the length of the CP (Cyclic Prefix) is higher than the length of the overall channel impulse response. We will assume that the $\mathrm{BS}$ has $L$ receive antennas and the received time-domain block associated to the $l$ th diversity branch, after discarding the samples associated to the $\mathrm{CP}$, is $\left\{y_{n}^{(l)} ; n=0,1, \ldots, N-1\right\}$. The corresponding frequency-domain block (i.e., the length- $N$ DFT (Discrete Fourier Transform) of the block $\left.\left\{y_{n}^{(l)} ; n=0,1, \ldots, N-1\right\}\right)$ is $\left\{Y_{k}^{(l)} ; k=0,1, \ldots, N-1\right\}$.

dimensions $K \times M$.
Let us consider first a linear transmitter. In this case, the frequency-domain block transmitted by the $p$ th MT is $\left\{S_{k, p}^{T x}=\right.$ $\left.S_{k, p} ; k=0,1, \ldots, N^{\prime}-1\right\}$ and

$$
\begin{aligned}
Y_{k}^{(l)} & =\sum_{p=1}^{P} S_{k, p} H_{k, p}^{C h(l)}+N_{k}^{(l)}= \\
& =\sum_{p=1}^{P} A_{k \operatorname{Mod} M, p} C_{k, p} \xi_{p} H_{k, p}^{C h(l)}+N_{k}^{(l)}= \\
& =\sum_{p=1}^{P} A_{k \operatorname{Mod} M, p} H_{k, p}^{(l)}+N_{k}^{(l)}
\end{aligned}
$$

with $H_{k, p}^{C h(l)}$ denoting the channel frequency response between the $p$ th MT and the $l$ th diversity branch, at the $k$ th subcarrier, $N_{k}^{(l)}$ the corresponding channel noise and $H_{k, p}^{(l)}=$ $\xi_{p} H_{k, p}^{C h(l)} C_{k, p}$. To detect the $k$ th symbol of the $p$ th MT we will use the set of subcarriers $\Psi_{k}=\{k, k+M, \ldots, k+(K-1) M\}$.

By defining $\mathbf{Y}(k)=\left[\mathbf{Y}^{(1)}(k) \cdots \mathbf{Y}^{(L)}(k)\right]^{T}$, with $\mathbf{Y}^{(l)}(k)$ denoting the line vector with the received samples associated to the set of frequencies $\Psi_{k}$, for the $l$ th antenna, and $\mathbf{A}(k)=$ $\left[A_{k, 1} \ldots A_{k, P}\right]^{T}$, we have

$$
\mathbf{Y}(k)=\mathbf{H}^{T}(k) \mathbf{A}(k)+\mathbf{N}(k)
$$

$\left((\cdot)^{T}\right.$ denote the transpose matrix), where $\mathbf{N}(k)=$ $\left[\mathbf{N}^{(1)}(k) \cdots \mathbf{N}^{(L)}(k)\right]^{T}$, with $\mathbf{N}^{(l)}(k)$ denoting the line vector with the noise samples associated to the set of frequencies $\Psi_{k}$, for the $l$ th antenna. In (4), $\mathbf{H}(k)$ is the overall channel matrix associated to $\mathbf{A}(k)$, i.e., $\mathbf{H}(k)=\left[\mathbf{H}^{(1)}(k) \cdots \mathbf{H}^{(L)}(k)\right]$, with $\mathbf{H}^{(l)}(k)$ denoting a $(P \times K)$ matrix, with lines associated to the different MTs and columns associated to the set of frequencies $\Psi_{k}$, for the $l$ th antenna.

We consider an iterative multiuser receiver with PIC (Parallel Interference Cancelation). The receiver can be described as follows. For a given iteration, the detection of $\mathbf{A}(k)$ employs the structure depicted in fig. 2, where we have $L$ feedforward filters (one for each receive antennas) and $P$ feedback loops. The feedforward filters are designed to minimize the MAI (Multiple Access Interference) that cannot be canceled by the feedback loops. For the first iteration we do not have any information about the MT's symbols and the receiver reduces to a linear multiuser receiver.

For each iteration, the samples associated to $\mathbf{A}(k), \tilde{\mathbf{A}}(k)$ are given by

$$
\tilde{\mathbf{A}}(k)=\mathbf{F}^{T}(k) \mathbf{Y}(k)-\mathbf{B}^{T}(k) \overline{\mathbf{A}}(k)
$$

where $\tilde{\mathbf{A}}(k)$ is defined as $\mathbf{A}(k), \mathbf{F}(k)=\left[\mathbf{F}^{(1)}(k) \cdots \mathbf{F}^{(L)}(k)\right]^{T}$ is the matrix of the feedforward filters' coefficients, with $\mathbf{F}^{(l)}(k)$ denoting a $(P \times K)$ matrix, with lines associated to 


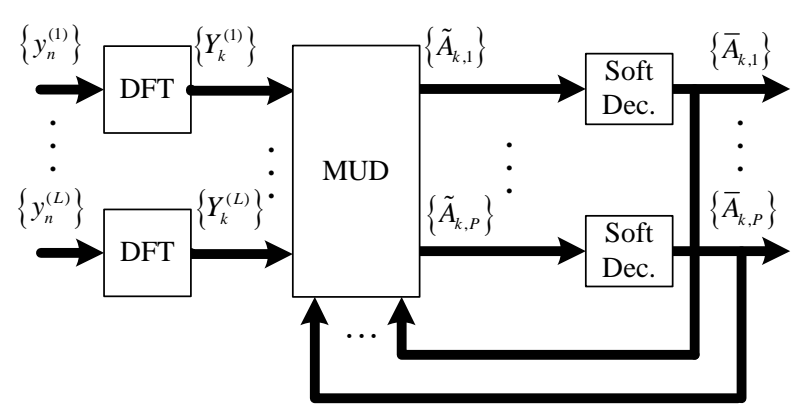

Figure 2: Iterative receiver for a linear transmitter.

the different MTs and columns associated to the set of frequencies $\Psi_{k}$, for the $l$ th antenna, and $\mathbf{B}(k)$ is a $P \times P$ matrix with the feedback filters' coefficients. $\overline{\mathbf{A}}(k)$, also defined as $\mathbf{A}(k)$, is the "average" value of $\mathbf{A}(k)$ from the previous iteration, which can be computed as follows.

Let us assume that the transmitted symbols are selected from a QPSK constellation under a Gray mapping rule (the generalization to other cases is straightforward). We will define $A_{k, p}= \pm 1 \pm j=A_{k, p}^{I}+j A_{k, p}^{Q}$, with $A_{k, p}^{I}=\operatorname{Re}\left\{A_{k, p}\right\}$ and $A_{k, p}^{Q}=\operatorname{Im}\left\{A_{k, p}\right\}, k=0,1, \ldots, M-1$, (similar definitions can be made for $\tilde{A}_{k, p}, \hat{A}_{k, p}$ and $\left.\bar{A}_{k, p}\right)$.

The LLRs (LogLikelihood Ratios) of the "in-phase bit" and the "quadrature bit", associated to $A_{k, p}^{I}$ and $A_{k, p}^{Q}$, respectively, are given by

$$
L_{k, p}^{I}=\frac{2}{\sigma_{p}^{2}} \tilde{A}_{k, p}^{I}
$$

and

$$
L_{k, p}^{Q}=\frac{2}{\sigma_{p}^{2}} \tilde{A}_{k, p}^{Q}
$$

respectively, where

$$
\sigma_{p}^{2}=\frac{1}{2} E\left[\left|A_{k, p}-\tilde{A}_{k, p}\right|^{2}\right] \approx \frac{1}{2 M} \sum_{k=0}^{M-1} E\left[\left|\hat{A}_{k, p}-\tilde{A}_{k, p}\right|^{2}\right]
$$

with $\hat{A}_{k, p}$ denoting the "hard decisions" associated to $\tilde{A}_{k, p}$.

Under a Gaussian assumption, it can be shown that the average value of $A_{k, p}$ is

$$
\begin{array}{r}
\bar{A}_{k, p}=\tanh \left(\frac{L_{k, p}^{I}}{2}\right)+j \tanh \left(\frac{L_{k, p}^{Q}}{2}\right)= \\
=\rho_{k, p}^{I} \hat{A}_{k, p}^{I}+j \rho_{k, p}^{Q} \hat{A}_{k, p}^{Q}
\end{array}
$$

The hard decisions $\hat{A}_{k, p}^{I}= \pm 1$ and $\hat{A}_{k, p}^{Q}= \pm 1$ are defined according to the signs of $L_{k, p}^{I}$ and $L_{k, p}^{Q}$, respectively;

$$
\rho_{k, p}^{I}=\frac{E\left[A_{k, p}^{I} \hat{A}_{k, p}^{I}\right]}{E\left[\left|A_{k, p}^{I}\right|^{2}\right]}=\tanh \left(\frac{\left|L_{k, p}^{I}\right|}{2}\right)
$$

and

$$
\rho_{k, p}^{Q}=\frac{E\left[A_{k, p}^{Q} \hat{A}_{k, p}^{Q}\right]}{E\left[\left|A_{k, p}^{Q}\right|^{2}\right]}=\tanh \left(\frac{\left|L_{k, p}^{Q}\right|}{2}\right)
$$

can be regarded as the reliabilities associated to the "in-phase" and "quadrature" bits of the $k$ th symbol of the $p$ th MT (naturally, $0 \leq \rho_{k, p}^{I} \leq 1$ and $\left.0 \leq \rho_{k, p}^{Q} \leq 1\right)$. For the first iteration, $\rho_{k, p}^{I}=\rho_{k, p}^{Q}=0$ and $\bar{A}_{k, p}=0$; after some iterations and/or when the SNR is high, typically $\rho_{k, p}^{I} \approx 1$ and $\rho_{k, p}^{Q} \approx 1$, leading to $\bar{A}_{k, p} \approx \hat{A}_{k, p}$. We can also define the blockwise reliability

$$
\rho_{p}=\frac{1}{M} \sum_{k=0}^{M-1} \frac{E\left[A_{k, p}^{*} \hat{A}_{k, p}\right]}{E\left[\left|A_{k, p}\right|^{2}\right]}=\frac{1}{2 M} \sum_{k=0}^{M-1}\left(\rho_{k, p}^{I}+\rho_{k, p}^{Q}\right) .
$$

It can be shown that the optimum feedforward coefficients in the MMSE sense can be written as

$$
\mathbf{F}(k)=\mathbf{F}^{I}(k) \boldsymbol{\Gamma}^{-1}
$$

with $\boldsymbol{\Gamma}=\operatorname{diag}\left(\gamma_{1}, \ldots, \gamma_{P}\right)$, where

$$
\gamma_{p}=\sum_{k^{\prime} \in \Psi_{k}} \sum_{l=1}^{L} F_{k^{\prime}, p}^{(l)} H_{k^{\prime}, p}^{(l)}
$$

and

$$
\mathbf{F}^{I}(k)=\left[\mathbf{H}^{H}(k)\left(\mathbf{I}_{P}-\mathbf{P}^{2}\right) \mathbf{H}(k)+\beta \mathbf{I}_{K L}\right]^{-1} \mathbf{H}^{H}(k),
$$

$\left((\cdot)^{H}\right.$ denotes the Hermitian matrix and $\mathbf{I}_{X}$ being the $X$-by$X$ identity matrix), with $\mathbf{P}=\operatorname{diag}\left(\rho_{1}, \ldots, \rho_{P}\right)$ and $\beta=$ $E\left[\left|N_{k}^{(l)}\right|^{2}\right] / E\left[\left|A_{k, p}\right|^{2}\right]$.

The optimum feedback coefficients are given by

$$
\mathbf{B}(k)=\mathbf{H}(k) \mathbf{F}(k)-\mathbf{I}_{P} .
$$

If we do not have data estimates for the different MTs, $\rho_{p}=$ $0(p=1,2, \ldots, P)$, and the feedback coefficients are zero. Therefore, (5) reduces to

$$
\tilde{\mathbf{A}}(k)=\mathbf{F}^{T}(k) \mathbf{Y}(k),
$$

which corresponds to the linear receiver.

It can be shown that the optimum feedforward coefficients can be written in the form

$$
\mathbf{F}(k)=\mathbf{H}^{H}(k) \mathbf{C}(k),
$$

apart a normalization factor as in (13), with $\mathbf{C}(k)$ given by

$$
\mathbf{C}(k)=\left[\left(\mathbf{I}_{P}-\mathbf{P}^{2}\right) \mathbf{H}(k) \mathbf{H}^{H}(k)+\beta \mathbf{I}_{P}\right]^{-1} .
$$

The computation of the feedforward coefficients from (18) is simpler than the direct computation, from (15), especially when $P<K$ and/or $L>1$.

\section{B. Nonlinear Transmitters}

It was shown in $[13,14]$ that we can improve significantly the performance of OFDM schemes submitted to nonlinear devices by employing a receiver with iterative cancelation of nonlinear distortion effects. This concept can be extended to MC-CDMA, leading to the receiver structure of fig. 3. The basic idea behind this receiver is to use an estimate of the 


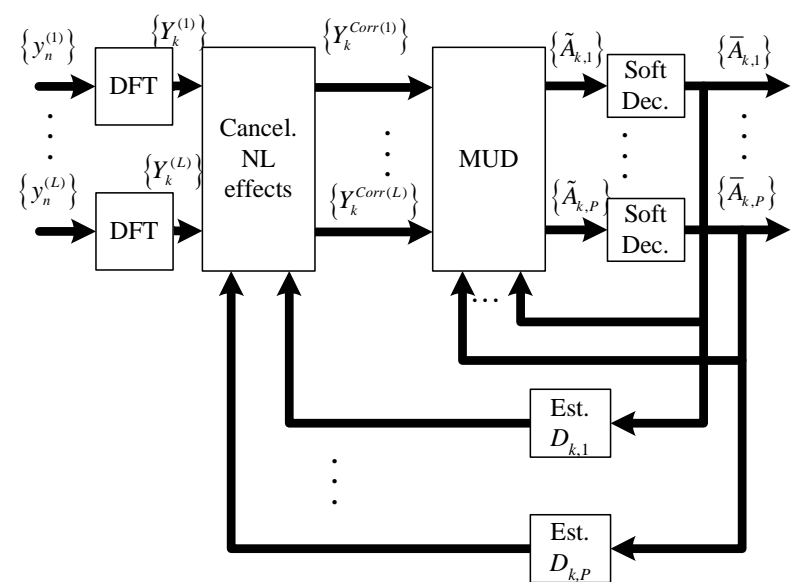

Figure 3: Iterative receiver with cancelation of nonlinear distortion effects.

nonlinear self-distortion $\left\{\bar{D}_{k, p} ; k=0,1, \ldots, N-1\right\}$ provided by the preceding iteration to remove the nonlinear distortion effects in the received samples. Therefore, the received frequency-domain block associated to the $l$ th diversity antenna, $\left\{Y_{k}^{(l)} ; k=0,1, \ldots, N-1\right\}$, is replaced by the corrected block $\left\{Y_{k}^{\operatorname{Corr}(l)} ; k=0,1, \ldots, N-1\right\}$, where

$$
Y_{k}^{C o r r(l)}=\frac{1}{\alpha_{p}}\left(Y_{k}^{(l)}-\sum_{p=1}^{P} H_{k, p}^{C h(l)} \bar{D}_{k, p}\right) .
$$

The remaining of the receiver is similar, but with $\beta$ given by

$$
\beta=\frac{E\left[\left|N_{k}^{(l)}\right|^{2}\right]}{E\left[\left|\alpha_{p} A_{k, p}\right|^{2}\right]+E\left[\left|D_{k, p}^{e q}\right|^{2}\right]},
$$

where

$$
D_{k, p}^{e q}=\frac{1}{K} \sum_{k^{\prime} \in \Psi_{k}} D_{k^{\prime}, p} C_{k^{\prime}, p}
$$

For the first iteration, the expectations of (21) can easily be obtained by using the analytical approach of [11, 12]; for the remaining iterations they have to be obtained by simulation. However, in most cases of interest we cancel a significant part of the nonlinear self-distortion at the first iteration; therefore, after the first iteration, we can ignore $E\left[\left|D_{k, p}^{e q}\right|^{2}\right]$ in (21).

For a given iteration, $\left\{\bar{D}_{k, p} ; k=0,1, \ldots, N-1\right\}$ can be estimated from $\left\{\bar{A}_{k, p} ; k=0,1, \ldots, M-1\right\}$ as follows: $\left\{\bar{A}_{k, p} ; k=0,1, \ldots, M-1\right\}$ is re-spread to generate the "average block to be transmitted" $\left\{\bar{S}_{k, p} ; k=0,1, \ldots, N-1\right\}$; $\left\{\bar{S}_{k, p} ; k=0,1, \ldots, N-1\right\}$ is submitted to a replica of the nonlinear signal processing scheme employed in the $p$ th transmitter so as to from the "average transmitted block" $\left\{\bar{S}_{k, p}^{T x} ; k=\right.$ $0,1, \ldots, N-1\} ; \bar{D}_{k, p}$ is given by

$$
\bar{D}_{k, p}=\bar{S}_{k, p}^{T x}-\alpha_{p} \bar{S}_{k, p}
$$

(naturally, for the first iteration, $\bar{D}_{k, p}=0$ ).

\section{PERformance Results}

In this section we present a set of performance results concerning the iterative receiver structures proposed in this paper for the uplink of MC-CDMA systems with frequency-domain spreading. The spreading factor is $K=16$ and we have $M=16$ data symbols for each user, corresponding to blocks with length $K M=256$, plus an appropriate CP. QPSK constellations, with Gray mapping, are employed. To reduce the envelope fluctuations (and the PMEPR) of the transmitted signals while maintaining the spectral occupation of conventional MC-CDMA schemes, each MT employs the clipping techniques combined with a frequency-domain filtering proposed in [11] (the power amplifiers are assumed to be linear for the (reduced) dynamic range of the envelope fluctuations of the transmitted signals). The receiver (i.e., the BS) knows the characteristics of the PMEPR-reducing signal processing technique employed by each MT.

We consider the power delay profile type $\mathrm{C}$ for the HIPERLAN/2 (HIgh PERformance Local Area Network) [15], with uncorrelated Rayleigh fading for the different MTs and for the different paths (similar results were obtained for other severely time-dispersive channels). The duration of the useful part of the block is $4 \mu \mathrm{s}$ and the $\mathrm{CP}$ has duration $1.25 \mu \mathrm{s}$. We consider uncoded BER performances under perfect synchronization and channel estimation conditions ${ }^{3}$. We have $P=K=16$ MTs, corresponding to a fully loaded scenario and $\xi_{p}=1$ for all MTs, i.e., we have a perfect power control (our simulations showed that the iterative receivers considered here are still suitable for scenarios without power control). At the BS we have $L$ uncorrelated receive antennas, for diversity purposes.

Let us first assume that we have linear transmitters at each MT. Fig. 4 shows the BER performance for each iteration (averaged over all MTs) when different values of $L$ are considered (naturally, the first iteration corresponds to a linear receiver). For the sake of comparisons, we also include the SU (SingleUser) performance, which, for the $k$ th data symbol could be defined as

$$
P_{b, S U, k}=E\left[Q\left(\sqrt{\frac{2 E_{b}}{N_{0}} \frac{1}{K} \sum_{k^{\prime} \in \Psi_{k}}\left|H_{k^{\prime}}\right|^{2}}\right)\right],
$$

where the expectation is over the set of channel realizations (it is assumed that $E\left[\left|H_{k}\right|^{2}\right]=1$ for any $k$ ).

From fig. 4 it is clear that the iterative receiver allows significant performance improvements relatively to the linear receiver. Moreover, we can be very close to the SU performance, especially when the diversity order is high.

Let us assume now that the normalized clipping level, identical to all MTs, is $s_{M} / \sigma=2.0$. This allows PMEPRs between $3.5 \mathrm{~dB}$ and $5.7 \mathrm{~dB}$, depending on the out-of-band radiation levels (if we want to maintain the spectral occupation of conventional MC-CDMA signals the PMEPR is $5.7 \mathrm{~dB}$ ) [11] (for conventional MC-CDMA signals with a large number of subcarriers the PMEPR is about $8.4 \mathrm{~dB}$ ). Fig. 5 shows the corresponding BER performances. Clearly, the performance of the linear receiver is very poor, with high irreducible error floors due to the nonlinear distortion effects. This is especially serious for the

\footnotetext{
${ }^{3}$ It should be noted that perfect time synchronization between the blocks associated to different MTs is not required since some time mismatches can be absorbed by the CP.
} 


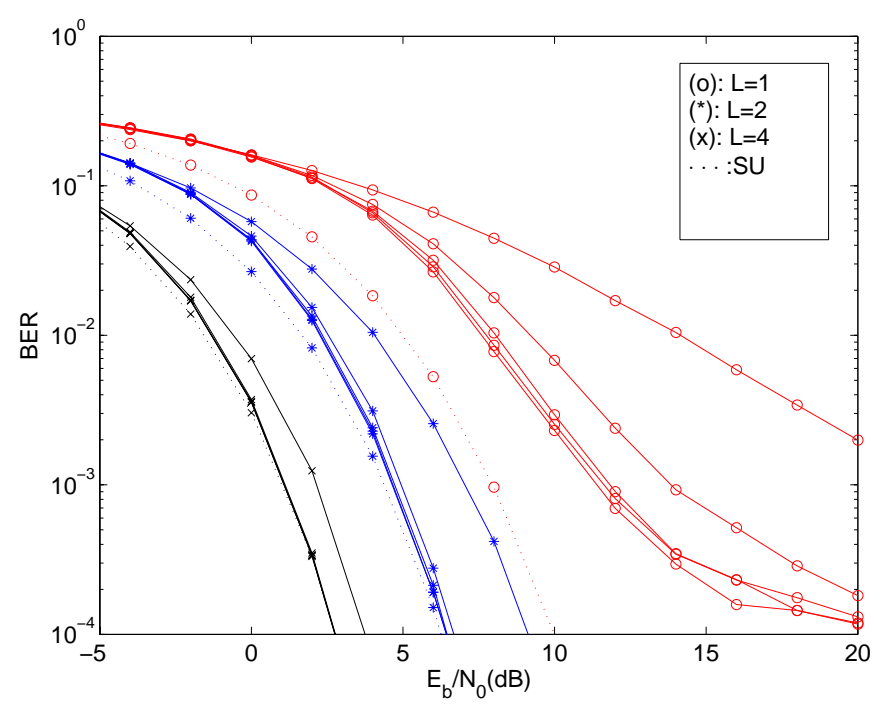

Figure 4: Average BER performance for iterations 1 to 5 (better performances as we increase the number of iterations), as well as the corresponding SU performance, when linear transmitters are considered.

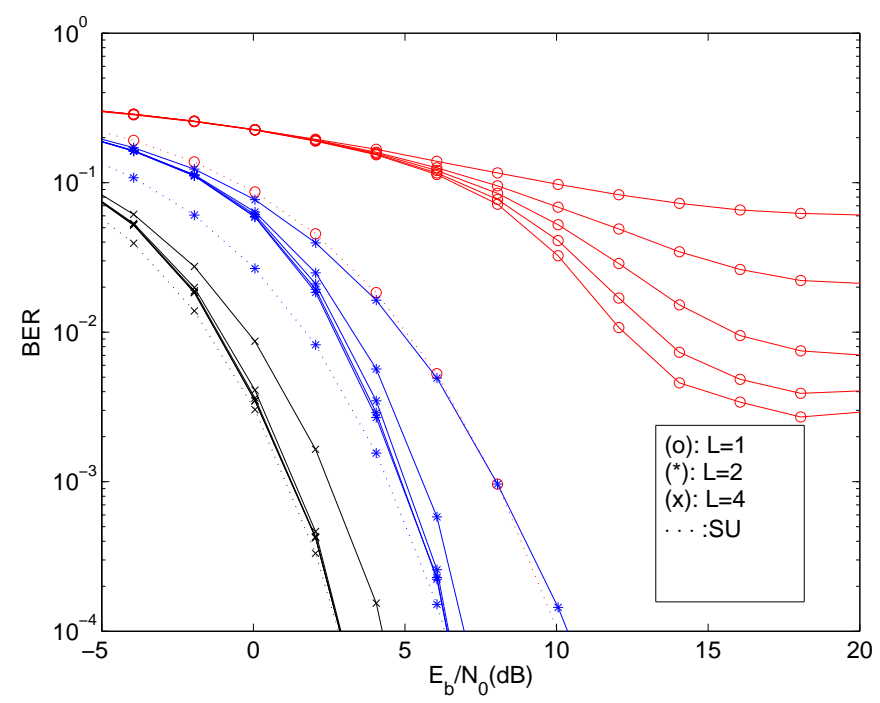

Figure 5: As in fig. 4, but for nonlinear transmitters.

case where $L=1$. As we increase the number of iterations and/or we increase $L$ improve significantly the performances, that can be close to the ones obtained with linear transmitters if $L>1$.

\section{CONCLUSIONS}

In this paper we considered the uplink transmission in MCCDMA systems. To allow an efficient power amplification, the MC-CDMA signal associated to each MT was submitted to a clipping device, followed by a frequency-domain filtering operation. We proposed an iterative receiver that jointly performs the MUD and the estimation and cancelation of the nonlinear distortion effects that are inherent to the transmitted signals.

Our performance results showed that the proposed receiver structures allow good performances, even for severely timedispersive channels and/or when a low-PMEPR is intended for each MT. The performances could be further improved if the feedback loop uses the channel decoder outputs instead of the MUD outputs, as suggested for OFDM schemes in [16]. This is especially important if we decrease the clipping level, to reduce further the PMEPR of the transmitted signals.

\section{REFERENCES}

[1] S. Hara and R. Prasad, "Overview of Multicarrier CDMA", IEEE Comm. Magazine, Dec. 1997.

[2] L.Cimini Jr., "Analysis and Simulation of a Digital Mobile Channel using Orthogonal Frequency Division Multiplexing", IEEE Trans. on Comm., Vol 33, No. 7, pp. 665-675, July, 1985.

[3] S. Hara and R. Prasad, "Design and Performance of Multicarrier CDMA System in Frequency-Selective Rayleigh Fading Channels", IEEE Trans. on Vehicular Technology, Vol. 48, No. 5, Sep. 1999.

[4] H. Sari, "Orthogonal Multicarrier CDMA and its Detection on Frequency-Selective Channels", European Trans. on Telecomm., Vol. 13, No. 5, pp. 439-445, Sep.-Oct. 2002.

[5] R. Dinis, P. Silva and A. Gusmão, "Iterative Block Decision Feedback Equalization for Multicarrier CDMA", IEEE VTC'05(Spring), Stockholm, Sweden, May 2005.

[6] N. Benvenuto and S. Tomasin, "Block Iterative DFE for Single Carrier Modulation", IEE Elec. Let., Vol. 39, No. 19, pp. 1144 1145, Sep. 2002.

[7] R. Dinis, A. Gusmão, and N. Esteves, "On Broadband Block Transmission over Strongly Frequency-Selective Fading Channels", Proc. Wireless 2003, Calgary, Canada, July 2003.

[8] S.Müller and J.Huber, "A Comparison of Peak Reduction Schemes for OFDM", IEEE GLOBECOM'97, Phoenix, 1997.

[9] X. Li and L. Cimini, "Effects of Clipping and Filtering on the Performance of OFDM", IEEE Comm. Letters, May 1998.

[10] P. Silva and R. Dinis, "A Technique for Reducing the PMEPR of MC-CDMA Signals", ECWT'04, Amsterdam, Oct. 2004.

[11] R. Dinis and A. Gusmão, "A Class of Nonlinear Signal Processing Schemes for Bandwidth-Efficient OFDM Transmission with Low Envelope Fluctuation”, IEEE Trans. on Comm., Vol. 52, No. 11, pp. 2009-2018, Nov. 2004.

[12] R. Dinis and P. Silva, "Analytical Evaluation of Nonlinear Effects in MC-CDMA Signals”, WOC'05, July 2005.

[13] J. Tellado, L. Hoo and J. Cioffi, "Maximum Likelihood Detection of Nonlinearly Distorted Multicarrier Symbols by Iterative Decoding”, IEEE Trans. on Comm. Vol. 51, Feb. 2003.

[14] A.Gusmão and R.Dinis, "Iterative Receiver Techniques for Cancellation of Deliberate Nonlinear Distortion in OFDM-type Transmission", Int. OFDM Workshop'04, Dresden, Sep. 2004.

[15] ETSI, "Channel models for HIPERLAN/2 in Different Indoor Scenarios”, ETSI EP BRAN 3ERI085B, pp. 1-8, March 1998.

[16] A. Gusmão, R. Dinis and P. Torres, "Low-PMEPR OFDM Transmission with an Iterative Receiver Technique for Cancellation of Nonlinear Distortion", IEEE VTC05(Fall), Sep. 2005. 